\title{
RSS-Based Localization with Different Antenna Orientations
}

\author{
B.J.Dil ${ }^{1}$, P.J.M.Havinga ${ }^{2}$ \\ Pervasive Systems, University of Twente \\ Zilverling building, 7522 NB Enschede, The Netherlands \\ ${ }^{1}$ B.J.Dil@utwente.nl \\ ${ }^{2}$ P.J.M.Havinga@ewi.utwente.nl \\ Ambient Systems \\ Colosseum 15d, 7521 PV Enschede, The Netherlands \\ ${ }^{1}$ Bram@ambient-systems.net
}

\begin{abstract}
This paper analyzes the influence of the antenna orientation on the performance of Received Signal Strength (RSS) based localization algorithms. Existing RSS-based localization algorithms provide reliable results in environments with static sources of error. This paper analyzes the performance of three RSS-based algorithms in an environment with the antenna orientation as a dynamic source of error. We first experimentally verify that the antenna orientation has a large influence on the received signal strength by performing an extensive amount of measurements. As expected, these measurements show that the signal strength may vary more than a factor five under different antenna orientations. This paper shows that antenna orientations may decrease the performance of optimally calibrated RSS-based localization algorithms by as much as $32 \%$, from 1.8 to 2.65 meter. In addition, it shows that improper calibration of the antenna orientation may decrease the accuracy by $64 \%$, from 1.9 to 3.1 meter.
\end{abstract}

\section{INTRODUCTION}

This paper focuses on localization in wireless networks. Localization in these networks describes the process of obtaining a physical location in an automated manner using wireless communication. Many wireless network applications rely on location information to perform their tasks. Locations provide context to the measured data (e.g. like measuring temperature); localization can be a stand-alone application (e.g. asset tracking in a distribution center) or provides support to the network service (e.g. routing). Today, such applications have evolved into real-time location systems (RTLS) using a wide range of wireless technologies. Many of these localization applications are based on Received Signal Strength (RSS) measurements, as RSS information is obtained without additional hardware and energy costs. Other localization systems use techniques like Time Difference Of Arrival (TDOA), Time Of Flight (TOF), Ultra Wide Band (UWB) and Angle Of Arrival (AOA). In general, these techniques are more accurate than RSSbased localization, but require specialized hardware, more processing, more communication and thus more energy (e.g. [10]).

This paper focuses on RSS-based localization. It analyzes the influence of the antenna orientation on the performance of existing Received Signal Strength (RSS) based localization algorithms ([3], [4] and [9]). Other examples that influence the signal strength are reflections, obstacles, temperature and humidity. Existing RSS-based localization algorithms use propagation models to account for these influences. The performance of these localization algorithms depend on how well the propagation model is able to capture these environmental influences. Figure 1 shows how existing RSS-based algorithms calibrate the propagation model. The calibration takes place in the "Calibrate nuisance parameters" phase. In this phase, the propagation model is calibrated on the basis of the calibration measurements. During the second phase, the "Localization" phase, the position of the node is estimated, both on the basis of the localization measurements as well as on the calibrated propagation model. Hence, existing RSS-based localization algorithms assume that the propagation model is calibrated before the "Localization" phase. This implies that these RSSbased algorithms provide the best results in environments with static sources of error. In this paper, we analyze and focus on the localization performance in environments with the antenna orientation as the dynamic sources of error. Existing work on calibration mainly focuses on:

- The difference between individual nodes (e.g. [5]). Individual differences between nodes remain largely static over time and can therefore be measured before localization.

- Different sources of error that influence the optimal propagation model settings as the antenna orientation (e.g. [11] and [12]). However, these articles do not evaluate any localization algorithms.

The main contributions of this work are:

- Quantify the influence of the antenna orientation on the signal strength and the propagation model. Measurements show that the signal strength may vary more than a factor 32 under different antenna orientations $(16 \mathrm{dBm})$.

- Quantify the influence of dynamic antenna orientations on the performance of existing RSS-based localization algorithms. Measurements show that the antenna orientation may decrease the performance of optimally calibrated 


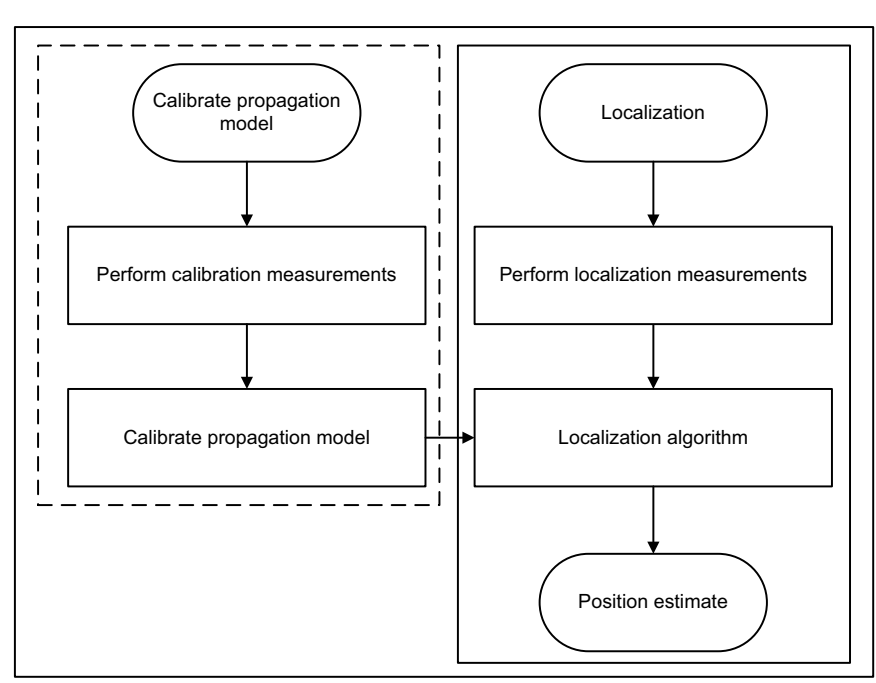

Fig. 1. Existing Localization Approach

RSS-based localization algorithms by as much as $32 \%$, from 1.8 to 2.65 meter.

- Quantify the influence of improperly calibrated propagation models on the accuracy of existing RSS-based localization algorithms. Measurements show that improperly calibrated algorithms may decrease the performance by as much as $64 \%$, from 1.9 to 3.1 meter.

This paper is organized as follows. After a short overview of existing RSS-based localization algorithms in Section II, we present the localization and measurement set-up in Section III. Section IV analyzes the influence of the antenna orientation on the received signal strength and quantifies the resulting changes in the propagation model. Section V analyzes the influence of the antenna orientation on the performance of existing RSS-based localization algorithms. Section VI summarizes the results.

\section{RELATED WORK}

This section provides a short overview of existing RSSbased localization methods. These localization methods are fingerprinting, range-based and proximity-based localization.

\section{- Fingerprinting:}

Many RSS-based localization systems make use of fingerprinting, first proposed by [3]. In the calibration phase, the signal strength is measured from static infrastructure nodes at several locations. The measurements, taken at a particular position, represent the fingerprint of that particular position. The localization area is thus divided into a large set of positions and assigned fingerprints. The stored fingerprints represent the parameters that are calibrated in the calibration phase. The localization phase consists of finding the closest match with the localization measurements in the database of fingerprints. Fingerprinting achieves a relatively high accuracy in static indoor

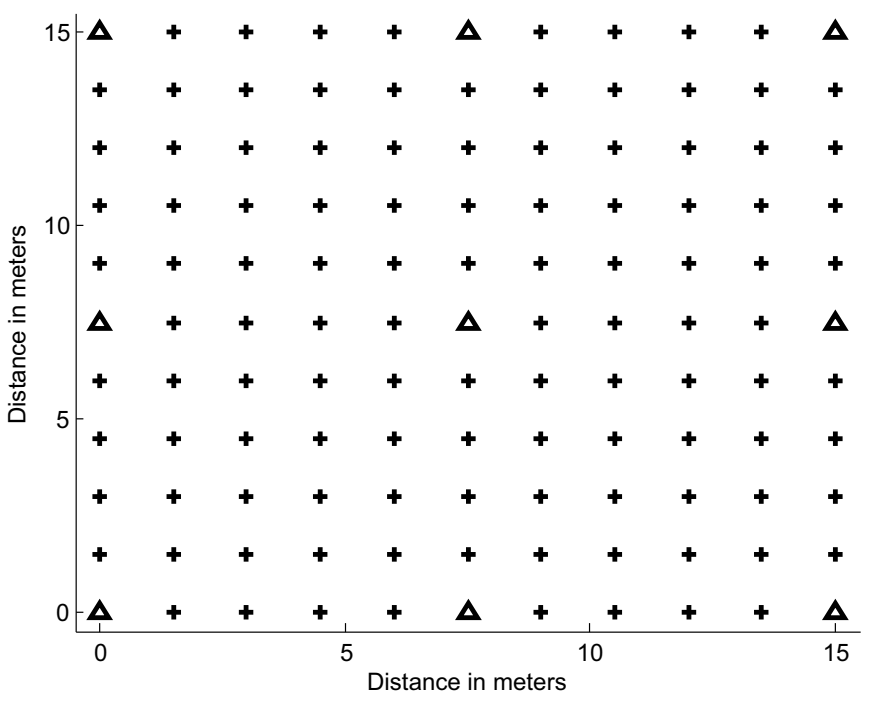

Fig. 2. Measurement set-up

environments, as it copes with static sources of noise such as walls that are common in indoor environments.

\section{- Range-based Localization:}

Range-based localization algorithms assume that the signal strength decay over distance follows a distribution that is known a priori. This distribution is used for converting one or several signal strength measurements into distance estimates. These distributions often include several parameters that try to capture the influence of the environment which are calibrated in the calibration phase (e.g. [4], [10] and [13]).

\section{- Proximity-based Localization:}

Proximity based localization algorithms assume that the signal strength decays with increasing distance ([6], [8] and [9]). The main difference with range-based algorithms is that proximity-based localization only uses the order of RSS measurements instead of converting signal strength to distance estimates. The advantage of proximity based localization algorithms is that they do not require a calibration phase.

This paper evaluates one RSS-based Fingerprinting-, Rangeand Promixity-based localization algorithm under dynamic antenna orientations.

\section{Localization And MEAsurement SET-UP}

This section first provides a formal description of the localization problem. After the problem formulation, this section provides a description of the measurement set-up. Consider a wireless network that consists of $N$ reference nodes and $M$ blind nodes:

- Reference nodes know their position in advance.

- Blind nodes do not know their location in advance.

This paper addresses the problem of positioning blind nodes using signal strength measurements from several reference nodes. We do not evaluate signal strength measurements 


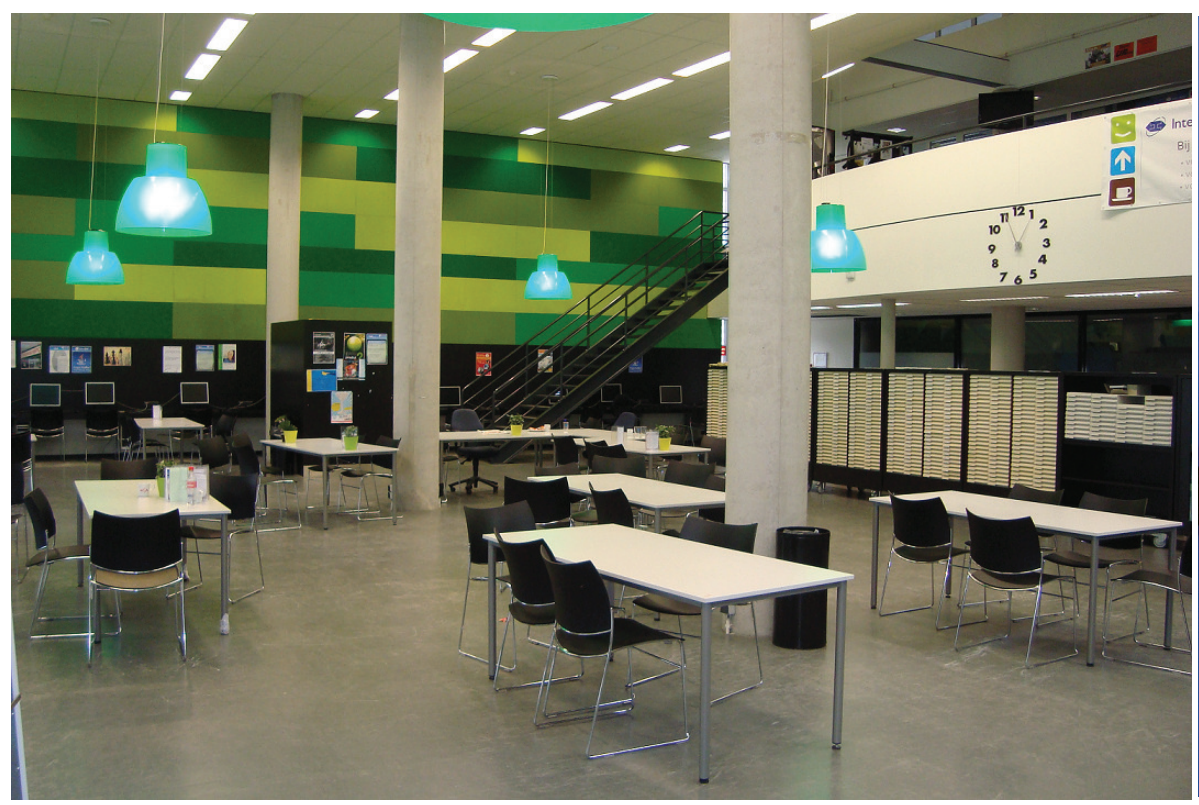

Fig. 3. Measurement environment

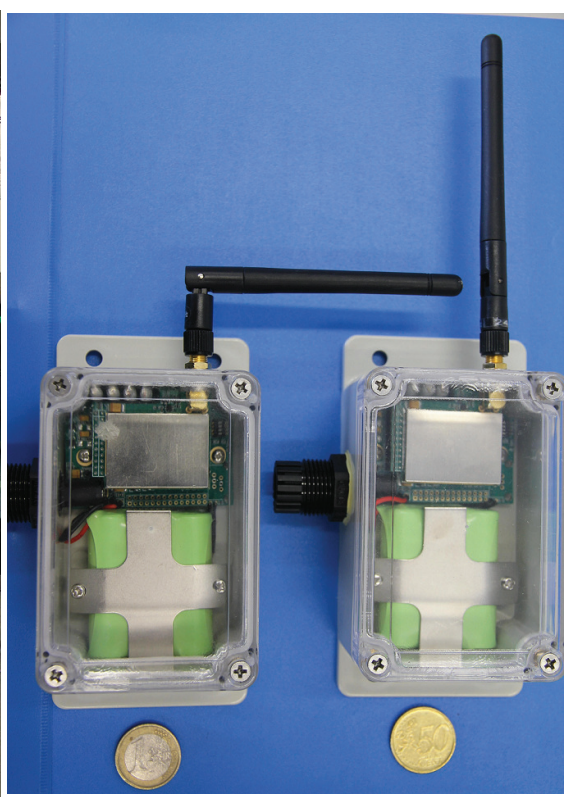

Fig. 4. Two CC2430 radio's between blind nodes (like in [4] and [10]).

Figure 2 shows the measurement set-up used throughout this paper. Here the nine triangles represent the reference node locations; the crosses represent the blind node locations. The measurements were conducted in an $15 \times 15$ meter open indoor study environment with ten CC2430 radios ([15]). We used nine CC2430 radios as reference nodes; these reference nodes were static during and between the measurement rounds (triangles). We used one CC2430 radio as blind node; this blind node measured the RSS at 112 different locations (crosses) to the reference nodes. The blind node measured 100 consecutive RSS measurements per frequency over a total of 38 frequencies and a bandwidth of 2408 . . $2480 \mathrm{MHz}$. Figure 3 shows the indoor environment and Figure 4 shows two CC2430 radios with casing. The reference nodes all had an "omnidirectional" dipole antenna with a vertical orientation. We performed two sets of measurement rounds on each location in which the blind node had a vertical or a horizontal antenna orientation (see Figure 4). The radios were placed at the same height of two meters in order to minimize the noise (e.g. [14]). All individual RSS measurements were sent to a computer and logged for post-processing. The conditions during the measurements were static (temperature, humidity, no moving objects). Therefore, we consider this environment as a static environment.

\section{Calibration Propagation Model}

This section describes the calibration of the propagation model on the basis of RSS measurements with different antenna orientations. We use the Log-Normal Shadowing Model (LNSM) for analyzing the signal strength over distance distribution. This model is widely used by RSS-based localization algorithms (e.g. [4], [10] and [13]) and has shown to be a reasonable representation of reality ([2]). The LogNormal Shadowing Model describes the signal strength decay over distance that suffers from shadowing effects. This model assumes that the received signal strength follows a log-normal distribution. Both theoretical and measurement-based studies support this assumption in indoor and outdoor environments ([2]). The following formula represents the Log-Normal Shadowing Model ([1]):

$$
P_{d}=P_{d_{0}}-10 \cdot n \cdot \log _{10}\left(\frac{d}{d_{0}}\right)+X_{\sigma_{d B m}}
$$

Here:

- $P_{d}$ represents the received signal strength in $d B m$ at distance $d$.

- $P_{d_{0}}$ represents the received signal strength in $d B m$ at reference distance $d_{0}$. In general distance $d_{0}$ is relatively small. For simplicity, we assume that distance $d_{0}$ is 1 meter (see [2]). $P_{d_{0}}$ depends on the transmission power and the gain of the sending and receiving antenna. Moreover, it depends on the polarization of the received signal relative to the orientation of the receiving antenna. This basically means that $P_{d_{0}}$ depends on the antenna orientation of the sender relative to the antenna orientation of the receiver. Throughout this paper, we refer to this variable by "Reference RSS".

- $n$ represents the Path Loss Exponent (PLE). The path loss exponent represents the rate at which the path loss increases with distance.

- $\sigma_{d B m}$ represents the standard deviation of the received signal strength due to shadowing effects and is invariant with the distance ([2]). $X_{\sigma_{d B m}}$ follows a zero-mean normal distribution with standard deviation $\sigma_{d B m}$ :

$$
X \sim N\left(0, \sigma_{d B m}^{2}\right)
$$




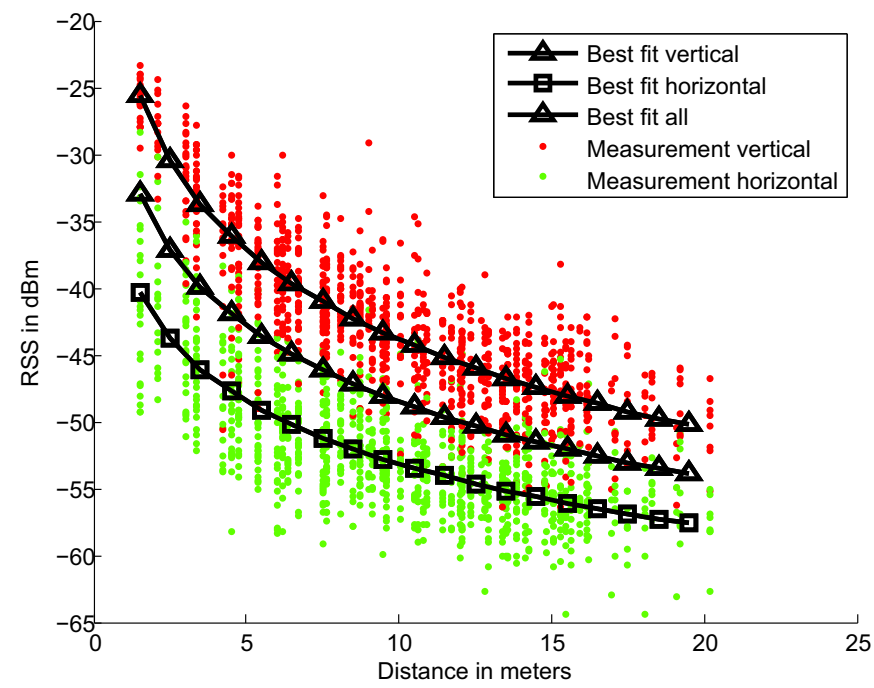

Fig. 5. RSS over distance distribution

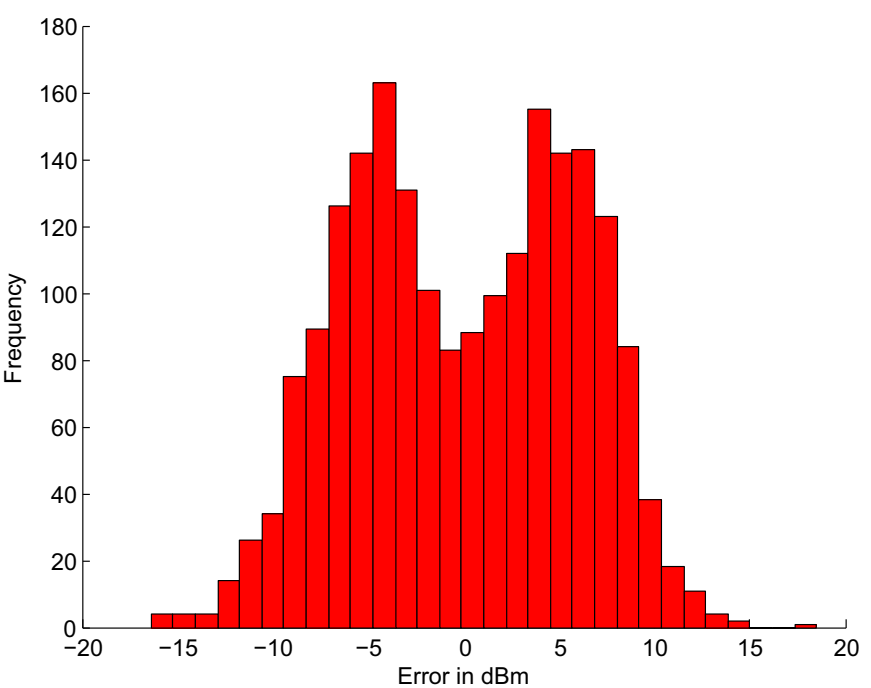

Fig. 6. Error distribution, log-normal shadowing model
The Log-Normal Shadowing Model (LNSM) is basically a scalar parametric model representing the electromagnetic field intensities from emitting, reflecting and refracting media in the so-called far-field zone. As long as those sources and media do not move or change, the parameters can be determined or calibrated using localization algorithms. In reality, emitters have directional radiation patterns that are polarized perpendicular to the direction of propagation. Media, including the receiving antennas, are generally sensitive to the polarization of the received signal strength. Hence, when the orientation of the antennas of the blind nodes is changing, the received signal strength changes. Hence, the Log-Normal Shadowing model assumes that the angle between the reference and blind node antenna remains equal.

Three major sources of error are multipath effects, shadowing ([1]) and hardware inaccuracies ([5]), assuming constant polarization effects. The multipath effect is usually minimized by performing RSS measurements over a large frequency bandwidth ([10]). The remaining errors are caused by the attenuation of the signal due to obstructions (shadowing). In our experimental set-up, we used line-of-sight measurements to ensure that the major sources of error would be multipath effects and hardware inaccuracies.

We performed two sets of measurement rounds on each location in which the blind node had a vertical or a horizontal antenna orientation. Figure 4 shows two nodes, one with a horizontal and one with a vertical antenna orientation. A sender/receiver pair with a vertical/vertical antenna orientation maximizes the received signal strength; A sender/receiver pair with perpendicular antenna orientations (vertical/horizontal) minimizes the received signal strength. Figure 5 shows the Log-Normal Shadowing Model fitting these real RSS measurements. The red dots represent individual RSS measurements with a vertical antenna orientation; the green dots represent individual RSS measurements with a horizontal antenna orien- tation. We distinguish three best fits that minimize the squared residual between the measured and expected RSS using the Log-Normal Shadowing Model, namely:

- The graph that fits the measurements made with a vertical antenna orientation. The parameter values of this fit are: $\left\{P_{d_{0}}=-21.6 \mathrm{dBm}, n=2.2, \sigma_{d B m}=3.2 \mathrm{dBm}\right\}$.

- The graph that fits the measurements made with a horizontal antenna orientation. The parameter values of this fit are: $\left\{P_{d_{0}}=-37.6 \mathrm{dBm}, n=1.5, \sigma_{d B m}=3.4 \mathrm{dBm}\right\}$.

- The graph that fits all measurements. The parameter values of this fit are: $\left\{P_{d_{0}}=-29.6 \mathrm{dBm}, n=1.9\right.$, $\left.\sigma_{d B m}=5.9 \mathrm{dBm}\right\}$.

The individual fits clearly show that even though we performed RSS measurements over a frequency bandwidth of $74 \mathrm{MHz}$, multipath effects are still significant sources of error.

Figure 6 shows the error distribution of the graph that fits all measurements. Figure 6 clearly shows that the error distribution consists of two normal distributions. This is because the graph that fits all measurements, fits two different LogNormal Shadowing models distinguished by the antenna orientations. The measurements verify that the RSS is significantly decreased by perpendicular antenna orientations, the reference $\operatorname{RSS}\left(P_{d_{0}}\right)$ is decreased by a factor five (from -21.6 to -37.6 ). Moreover, the measurements show that the path loss exponent is decreased by $32 \%$ (from 2.2 to 1.5 ). We expect that this is due to polarization effects.

\section{Performance of Localization Algorithms}

In this section we evaluate the performance of several RSS-based localization algorithms by performing an extensive amount of real measurements. Moreover, this section analyzes to what extent the performance depends on the calibration accuracy. We evaluate these algorithms in the set-up described in Section III, and we use the parameter values for the LogNormal Shadowing Model calculated in Section IV. This 


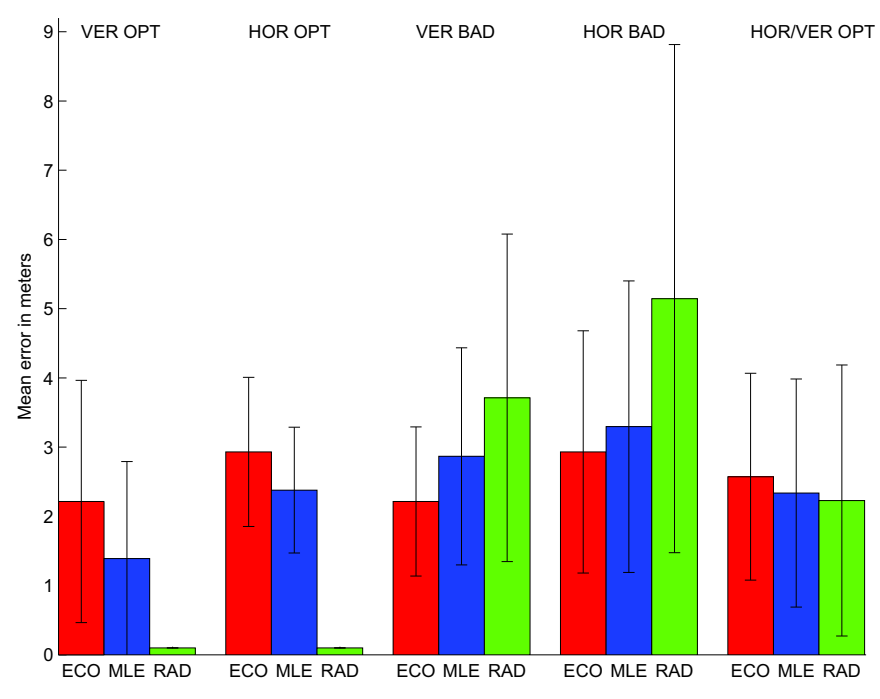

Fig. 7. Calibration and Performance real measurements

section compares the following RSS-based localization algorithms:

- Proximity-based localization algorithm: ECOLOCATION ([9], abbreviated by ECO). To our knowledge, ECOLOCATION provides the best performance of the RSS-based proximity localization algorithms ([9]).

- Range-based localization algorithm: The Maximum Likelihood Estimator based on the Log-Normal Shadowing model ([4], abbreviated by MLE). To our knowledge, [4] provides the best results given the Log-Normal Shadowing model (e.g. [4], [10] and [13]).

- Fingerprinting-based localization algorithm: RADAR ([3], abbreviated by RAD).

RADAR [3] put constraints on the position estimate, which increases the performance. Therefore, we also put the same constraints on the other localization algorithms in order to make a fair comparison. Note that we picked one localization algorithm per localization method described in Section II in order to characterize the performance per localization method.

\section{A. Calibration settings}

We consider the following calibration settings:

\section{- Optimal propagation model settings}

These propagation model settings represent the real and optimal propagation model settings that optimize the localization performance. The optimal propagation settings are a function of the localization measurements.

- Calibrated propagation model settings

The calibrated propagation model settings represent the propagation model settings that are used by the localization algorithms. The calibrated propagation settings are a function of the calibration measurements.

This means that the "Calibrated propagation model settings" are equal to the "Optimal propagation model settings" when the calibration measurements are equal to the localization

\begin{tabular}{|l|l|l|}
\hline Abbreviation & Localization measurements & Calibration measurements \\
\hline VER OPT & VER & VER \\
HOR OPT & HOR & HOR \\
VER BAD & VER & HOR \\
HOR BAD & HOR & VER \\
HOR/VER OPT & HOR/VER & HOR/VER \\
\hline
\end{tabular}

TABLE I

CALIBRATION APPROACHES

measurements. This section analyzes to what extent the performance depends on the calibration accuracy by using different calibration/localization measurement pairs. This section distinguishes between the same measurements as in Section IV (see Figure 5), namely the measurements made with a vertical/horizontal/vertical+horizontal blind node antenna orientation. We refer to these measurements by respectively "VER","HOR" and "HOR/VER". On the basis of these measurements, we distinguish between five calibration approaches as given in Table I.

\section{B. Performance}

Figure 7 shows the performance of the three localization algorithms associated with the five calibration approaches. The vertical lines represent the calculated standard deviations. Figure 7 shows that the performance of:

- ECO is independent of the localization/calibration measurement pair. This is logical because ECO does not require/use calibration measurements.

- MLE decreases with $63 \%$ with a badly calibrated propagation model ( $\sim 1.9$ to $\sim 3.1$ meter $)$.

- RAD decreases from $\sim 0$ to $\sim 4.4$ meter with a badly calibrated propagation model. Moreover, RAD estimates the real locations when the propagation model is optimally calibrated ("VER OPT" and "HOR OPT"). This is 


\begin{tabular}{|l|l|l|l|}
\hline method & Calibration Acc & Static Acc & Dynamic Acc \\
\hline Fingerprinting & -- & ++ & - \\
Range-based & - & + & - \\
Proximity & + & - & - \\
\hline
\end{tabular}

TABLE II

LOCALIZATION METHODS

logical because there is one localization measurement per blind node position per antenna orientation.

Figure 7 shows that the performance of ECO/MLE with "VER OPT/VER BAD" is significantly better than "HOR OPT/HOR BAD". This is because the error is inherent to the Log-Normal Shadowing model parameter settings. The error increases linearly with the following ratio ([7]): $\frac{X_{\sigma}}{n}$ and $\frac{3.4}{1.5}>\frac{3.2}{2.2}$.

MLE and RAD outperform ECO with optimally calibrated propagation models in environments with either a vertical or horizontal antenna orientation. However, this performance gain is decreased in an environment with both vertical and horizontal antenna orientations. Hence, Figure 7 shows that ECO provides similar results as MLE and RAD with "HOR/VER OPT". Moreover, ECO outperforms MLE and RAD when they are badly calibrated, which clearly shows the strength of ECO. Therefore, we expect that the performance of ECO approximates the performance of optimally calibrated MLE and RAD in a dynamic and thus more realistic environment. Table II provides an overview of the performance of the localization methods in relation to the following aspects:

- The Calibration Acc column represents to what extent the performance depends on the calibration measurements.

- The Static Acc column represents how well the localization method performs in environments with static sources of noise.

- The Dynamic Acc column represents how well the localization method performs in environments with dynamic sources of noise.

The "+"/“-" represents that the localization method scores $\operatorname{good} / \mathrm{bad}$ on this aspect.

\section{CONCLUSION}

In this study, we demonstrated that antenna orientation has a large influence on the RSS and the performance of existing RSS-based localization algorithms and methods by analyzing an extensive amount of RSS measurements. This large influence on received signal strengths is in line with the well-known polarization effects of electromagnetic radiation on media like antennas. We show that the calibration of the propagation model has a large influence on the localization performance. Optimally calibrated fingerprinting- and rangebased localization algorithms outperform proximity-based localization algorithms in an environment with one antenna orientation. This is the other way around when the propagation model is improperly calibrated. Moreover, proximity-based localization algorithms provide similar results as optimally calibrated fingerprinting- and range-based localization algorithms in an environment with two different antenna orientations. The antenna orientation is one of many environmental influences that influence the RSS and thus the localization performance. Therefore, we expect that proximity-based localization algorithms provide similar results as optimally calibrated fingerprinting- and range-based localization algorithms in more realistic environments with a variety of dynamic sources of error.

\section{ACKNOWLEDGEMENTS}

This paper describes work in part undertaken in the context of the SENSEI project, "Integrating the Physical with the Digital World of the Network of the Future" (www.senseiproject.eu). SENSEI is a Large Scale Collaborative Project supported by the European 7th Framework Programme, contract number: 215923.

\section{REFERENCES}

[1] Hashemi H.: The indoor radio propagation channel, Proc. IEEE, July 1993, pp. 943- 996.

[2] Rappaport T.S., Wireless Communication: Principles and Practice, Prentice Hall, ISBN 013 3755633, 1996

[3] P. Bahl and V. N. Padmanabhan: RADAR: An In-Building RF-Based User Location and Tracking System, in Proceedings of the 19th IEEE International Conference on Computer Communications (INFOCOM), March 2000

[4] N.Patwari, R.J.O’Dea, Y.Wang: Relative Location in Wireless Networks. Presented at IEEE Vehicular Technology Conference, Spring, Rhodes, Greece, May 2001.

[5] K.Whitehouse, D.Culler: Calibration as Parameter Estimation in Sensor Networks. In ACM International Workshop on Wireless Sensor Networks and Applications, Atlanta, GA, USA, September 2002.

[6] T.He, C.Huang, B.M.Blum, J.Stankovic, T.Abdelzaher: Range-free localization schemes for large scale sensor networks. MobiCom, San Diego, California, September 2003, pp. 81-95.

[7] N. Patwari, A.O. Hero, M.Perkins, N.S.Correal, R.J.O'Dea: Relative Location Estimation in Wireless Sensor Networks. IEEE Transactions on Signal Processing, special issue on Signal Processing in Networks, vol. 51, no. 8, August 2003, pp. 2137-2148.

[8] C.Liu, K.Wu, T.He: Sensor localization with ring overlapping based on comparison of received signal strength indicator, in IEEE Mobile Ad-hoc and Sensor Systems (MASS), Oct. 2004.

[9] K.Yedavalli, B.Krishnamachari, S.Ravula, and B.Srinivasan: Ecolocation: A sequence based technique for RF-only localization in wireless sensor networks. In IEEE IPSN 2005, April 2005.

[10] N.Patwari: Location estimation in sensor networks. Thesis of Neal Patwari at University of Michigan, 2005.

[11] D.Lymberopoulos, Q.Lindsey and A.Savvides: An Empirical Analysis of Radio Signal Strength Variability in IEEE 802.15.4 Networks using Monopole Antennas. Proceedings of the Second European Workshop on Sensor Networks (EWSN), 2006.

[12] T.Stoyanova, F.Kerasiotis, A.Prayatia and G.Papadopoulos: Evaluation of Impact Factors on RSS Accuracy for Localization and Tracking Applications. Proceedings of the 5th ACM international Workshop on Mobility Management and Wireless Access (Mobiwac), 2007.

[13] Rong Peng, Mihail L. Sichitiu: Probabilistic Localization for Outdoor Wireless Sensor Networks. ACM SIGMOBILE Mobile Computing and Communications, Volume 11, Issue 1, January 2007, pp. 53-64.

[14] K.Whitehouse, C.Karlof, D.Culler: A Practical Evaluation of Radio Signal Strength for Ranging-based Localization. Mobile Computing and Communications Review, Volume 11, Number 1, 2007.

[15] http://focus.ti.com/lit/ds/symlink/cc2430.pdf, 2010. 